\title{
Learning curve for gastric cancer surgery based on actual survival
}

\author{
Chan Young Kim • Byung-Ho Nam · Gyu Seok Cho $\cdot$ Woo Jin Hyung • \\ Min Chan Kim • Hyuk-Joon Lee $\cdot$ Keun Won Ryu • Sung Wan Ryu • \\ Dong Woo Shin · Jun Ho Lee
}

Received: 9 December 2014/ Accepted: 1 February 2015/Published online: 25 February 2015

(c) The International Gastric Cancer Association and The Japanese Gastric Cancer Association 2015

\begin{abstract}
Background The aim of this study was to investigate learning curves for surgeons performing D2 lymph node dissection based on actual patient survival.

Methods A total of 3,284 patients with gastric cancer who underwent curative intent gastric cancer surgery by nine surgeons in eight Korean hospitals between 2001 and 2006 were included. Each surgeon's experience was coded as the number of $\mathrm{D} 1+\beta$ or more gastrectomies performed before that for each patient, which indicates the surgeon's total number of prior surgical experiences. Surgeon experience was grouped into two sets of categories. The set of categories included four groups of experience: $\leq 50$,
\end{abstract}

C. Y. Kim · B.-H. Nam - G. S. Cho · W. J. Hyung ·

M. C. Kim - H.-J. Lee $\cdot$ K. W. Ryu $\cdot$ S. W. Ryu

D. W. Shin - J. H. Lee

Center for Gastric Cancer, Gastric Cancer Branch, National

Cancer Center, 809 Madu1-dong, Ilsandong-gu, Goyang,

Gyeonggi 411-769, Korea

e-mail: happyhill@chonbuk.ac.kr

B.-H. Nam

e-mail: byunghonam@ncc.re.kr

G. S. Cho

e-mail: gschogs@schbc.ac.kr

W. J. Hyung

e-mail: wjhyung@yumc.yonsei.ac.kr

M. C. Kim

e-mail: mckim@dau.ac.kr

H.-J. Lee

e-mail: appe98@snu.ac.kr

K. W. Ryu

e-mail: docryu@korea.com

S. W. Ryu

e-mail: gsman@dsmc.or.kr
51-100, 101-200, and >200 applicable operations. Multivariate survival time regression models were used to evaluate the association between surgeon experience and overall survival.

Results The learning curve for gastric cancer survival after open gastric cancer surgery was steep and did not reach a plateau until a surgeon completed 100 operations. Overall survival rate was the lowest among patients treated by a surgeon with an experience of 50-100 cases. The overall survival of patients at 5 years when the surgeon had a history of more than 100 experiences was higher in each stage than that when the surgeon had a history of fewer than 100 experiences.

D. W. Shin

e-mail: shin519@dmc.or.kr

C. Y. Kim

Department of Surgery, Chonbuk National University, Jeonju, Korea

B.-H. Nam

Center for Clinical Trial, National Cancer Center, Goyang,

Korea

G. S. Cho

Department of Surgery, Soonchunhayng University, Cheonan, Korea

W. J. Hyung

Department of Surgery, Yonsei University, Seoul, Korea

M. C. Kim

Department of Surgery, Dong-a University, Busan, Korea

H.-J. Lee

Department of Surgery, Seoul National University, Seoul, Korea 
Conclusion As a surgeon's experience increases, survival after gastric cancer surgery improves. Special attention needs to be paid to the second period of surgeon experience because survival of patients in this period was the lowest.

Keywords Gastric cancer - Surgery - Learning curve

\section{Introduction}

Despite decreasing incidence and mortality rates in developed countries, gastric cancer remains the sixth most commonly diagnosed cancer and the third most common cause of cancer mortality globally [1]. D2 lymph node dissection is recommended as a surgical approach for patients with curable gastric cancer [2-4] Two European randomized controlled trials (Dutch and MRC trials) that compared D1 and D2 lymph node dissection revealed that D2 lymph node dissection should be restricted to specialized centers where adequate training and supervision can be provided [5, 6].

The most important outcome of cancer surgery is survival. The outcome of surgery is generally thought to depend on the experience of the surgeon [7]. Actual learning curves based on survival data are rarely presented. Recent reports from prostate cancer have shown that as a surgeon's experience increases, cancer control after radical prostatectomy improves [8, 9]. However, differences in cases seen by surgeons might contribute to residual confounding findings, and the endpoint was not actual survival but instead biochemical recurrence in those studies; therefore, findings of related reports are of uncertain clinical relevance to patients.

Thus, the aim of the present study was to investigate learning curves for surgeons performing D2 lymph node dissection based on actual survival. In this study, we could observe a trend of learning curves in cancer surgery.

K. W. Ryu

Gastric Cancer Branch, National Cancer Center, Goyang, Korea

S. W. Ryu

Department of Surgery, Kemyeong University, Daegu, Korea

D. W. Shin

Department of Surgery, Jaeseng Hospital, Seongnam, Korea

Present Address:

J. H. Lee $(\bowtie)$

Department of Surgery, Sungkyunkwan University School of

Medicine, Seoul, Republic of Korea

e-mail: gsjunholee@gmail.com

\section{Patients and methods}

Study cohort and data sources

The study cohort consisted of 3,284 patients with gastric cancer who were treated by open gastric cancer surgery between January 2001 and December 2006 at one of eight institutions in Korea: Chonbuk National University, Donga University, Jaeseng Hospital, Kemyeong University, Seoul National University, Soonchunhayng University, Yonsei University, and National Cancer Center. All patients were treated by participating surgeons as a staff surgeon. Patients who (1) had a histologically confirmed gastric adenocarcinoma and (2) had newly diagnosed cancer without previous treatment were included in the analysis. Patients who underwent $\mathrm{R} 2$ resection $(n=122)$ or who underwent exploratory laparotomy only or bypass surgery $(n=102)$ were excluded. Finally, 3,060 patients who underwent potentially curative resection for gastric cancer were included in this study. All information was obtained with appropriate Institutional Review Board waivers, and data were collected without revealing any personal information.

\section{Personal characteristics and clinical data}

All characteristics of patients were obtained from a review of medical records. Demographic characteristics included age, sex, and body mass index. Clinicopathological characteristics included tumor location, tumor size, differentiation, Lauren classification, gross type, depth of invasion, lymph node metastasis, stage at diagnosis, operation method, and extent of lymph node dissection. Pathological diagnosis was determined with adherence to the sixth edition of the Union International Centre Cancer (UICC)/American Joint Committee on Cancer classification system [10]. In patients with multiple synchronous gastric cancers, the lesion with the deepest infiltration of the gastric wall was regarded as the main lesion and any others were regarded as accessory lesions. The clinicopathological characteristics of the main lesion were used for the analysis.

\section{Requirements for surgeon}

Each participating surgeon had an appointment as a staff surgeon during the study period, in which each surgeon had to perform at least 150 cases of open gastric cancer surgery to be eligible. Before the study period, all surgeons had completed a 4-year-long residency course on general surgery at a domestic teaching hospital followed by a 2-yearlong fellowship course specific to gastric cancer surgery. 
The fellowship course had to be attended at the institutions where the surgeons resumed their career as a staff surgeon during the study period. Surgeons who had previously conducted gastric cancer surgeries either as a fellow or as a staff surgeon at other institutions were not included in this study because of difficulties in accurate data collection. Eligible patients were treated by one of nine surgeons, all of whom saw patients only at a study institution while on the staff there.

\section{Operative procedures}

The surgical procedures and reconstruction methods were as follows. Gastrectomy was performed if a tumor-free margin of $4 \mathrm{~cm}$ was present in cases with advanced gastric carcinoma or of $2 \mathrm{~cm}$ in cases with early gastric carcinoma. Extent of lymph node dissection was determined using the recommendations of the Japanese Research Society for Gastric Carcinoma [11]. After laparotomy, surgeons examined intraabdominal cavities and inspected the peritoneum, diaphragm, liver capsule, and pelvic cavity. All patients enrolled in the present study underwent gastrectomy with $\mathrm{D} 1+\beta$ or more lymph node dissection [11]. Patients with early gastric cancer underwent D1 $+\beta$ lymph node dissection and those with advanced gastric cancer underwent D2 lymph node dissection. Tumors were staged in accordance with the 6th UICC tumor node metastasis (TNM) classification [10].

\section{Chemotherapy}

Not all patients were given postoperative chemotherapy. The indication for adjuvant treatment was not standardized among the various institutions. However, most patients with advanced gastric cancer more than TNM stage I were given 5-fluorouracil-based postoperative chemotherapy [12]. No patients were given postoperative or preoperative radiotherapy.

\section{Follow-up schedule}

Patient follow-up was conducted according to accepted clinical practice procedures at each institution. In general, follow-up consisted of an abdominopelvic computed tomogram every 6 months for 5 years after surgery and esophagogastro-duodenoscopy annually for 5 years after surgery. Cancer recurrence was defined as positive radiologic evidence.

Statistical methods and analyses

Follow-up of patients was completed until death or until the cutoff date of December 30, 2010. At the time of the last follow-up, 97 patients ( $3.2 \%$ ) had been lost to followup. The median follow-up interval for 3,060 patients alive at the cutoff date was 52 months (range, 0-106 months). The lost cases were treated as censored data for the analysis of survival rates. Overall survival was defined as the time from surgery to death (resulting from any cause). The Kaplan-Meier method [13] was used for estimating survival. The difference between survival curves was assessed using the log-rank test. The accepted level of statistical significance was $P<0.05$.

Each surgeon's experience was coded as the number of $\mathrm{D} 1+\beta$ or more gastrectomies performed before each patient, which indicates the surgeon's total number of prior surgical experiences. Surgeon experience was grouped into two sets of categories, which included four groups of experience $(\leq 50,51-100,101-200$, and $>200$ applicable operations).

To generate surgical learning curves, a stratified Cox regression model was used. A quadratic function of surgical experience was used in the model. The median predicted survival probability was calculated from the model for each level of experience.

\section{Results}

Patient characteristics by surgeon experience

Clinical and pathological characteristics of the tumors are shown in Table 1. The median age of the 3,060 patients was 54 years (range, 20-91 years). Of the total patients, 2,113 (69.1\%) were men and 947 (30.9\%) were women. In terms of location, 1,662 gastric cancers $(54.3 \%)$ were in the lower one third of the stomach and the other 1,398 cancers were located in the middle or upper one third of the stomach. Among the cancers, 1,275 cancers $(41.7 \%)$ were early gastric cancers. Clinical and pathological characteristics were comparable throughout the periods.

\section{Surgical outcomes by surgeon experience}

Surgical outcomes based on the number of surgical experiences are summarized in Table 2. Two of nine surgeons had performed fewer than 200 cases. As surgical experience increased, operating times $(t$ test, $P<0.001)$ and duration of hospital stay ( $t$ test, $P=0.001$ ) were shortened and the number of dissected lymph nodes ( $t$ test, $P<0.001)$ was increased. The rate of R0 resection was the lowest at the second period ( $\chi^{2}$ test, $\left.P=0.011\right)$. R1 resection represented cases of positive resection margin. Postoperative mortalities were not different throughout the periods ( $\chi^{2}$ test, $P=0.237$ ). 
Table 1 Patient characteristics

\begin{tabular}{|c|c|c|c|c|c|}
\hline $\begin{array}{l}\text { Surgeon experience (number of gastrectomies before the } \\
\text { incident case) }\end{array}$ & $\begin{array}{l}<51 n=450 \\
(\%)\end{array}$ & $\begin{array}{l}51-100 n=450 \\
(\%)\end{array}$ & $\begin{array}{l}101-200 n=814 \\
(\%)\end{array}$ & $\begin{array}{l}>200 n=1346 \\
(\%)\end{array}$ & $P$ value \\
\hline Sex & & & & & 0.34 \\
\hline Male & $305(67.8)$ & $300(66.7)$ & $557(68.4)$ & $952(70.7)$ & \\
\hline Female & $145(32.2)$ & $150(33.3)$ & 257 (31.6) & $395(29.3)$ & \\
\hline Age (years, mean $\pm \mathrm{SD}$ ) & $58.4 \pm 11.5$ & $58.8 \pm 11.5$ & $58.2 \pm 11.9$ & $58.4 \pm 12.0$ & 0.87 \\
\hline Body mass index $\left(\mathrm{kg} / \mathrm{m}^{2}\right.$, mean $\left.\pm \mathrm{SD}\right)$ & $22.9 \pm 11.5$ & $23.0 \pm 3.1$ & $23.5 \pm 13.8$ & $23.2 \pm 3.2$ & 0.51 \\
\hline Location of tumor & & & & & 0.83 \\
\hline Upper one third & 63.(13.5) & $56(12.4)$ & $119(14.6)$ & $228(16.9)$ & \\
\hline Mid one third & $112(24.9)$ & $131(29.1)$ & $228(28.0)$ & $397(29.5)$ & \\
\hline Lower one third & $266(59.1)$ & $251(55.8)$ & $453(55.7)$ & $692(51.4)$ & \\
\hline Whole & $9(2.0)$ & $12(2.6)$ & $14(1.7)$ & $29(2.2)$ & \\
\hline Tumor size $(\mathrm{cm}$, mean $\pm \mathrm{SD})$ & $4.8 \pm 3.3$ & $5.0 \pm 3.5$ & $4.8 \pm 3.0$ & $5.0 \pm 3.2$ & \\
\hline Type of gastric resection & & & & & 0.20 \\
\hline Subtotal & $329(73.1)$ & $310(68.9)$ & $588(72.2)$ & $929(69.0)$ & \\
\hline Total & $121(26.9)$ & $140(31.1)$ & $226(27.8)$ & $417(31.0)$ & \\
\hline Differentiation of tumor & & & & & 0.69 \\
\hline Differentiated & $183(40.7)$ & $198(44.1)$ & $335(41.2)$ & $572(42.5)$ & \\
\hline Undifferentiated & $267(59.3)$ & $251(55.9)$ & $478(58.8)$ & 417 (57.7) & \\
\hline TNM stages & & & & & 0.35 \\
\hline Stage I & $208(46.2)$ & $204(45.3)$ & $368(45.2)$ & $614(45.6)$ & \\
\hline Stage II & $77(17.1)$ & $72(16.0)$ & $181(22.2)$ & $270(20.1)$ & \\
\hline Stage III & $151(33.6)$ & $161(35.8)$ & $242(29.7)$ & $430(31.9)$ & \\
\hline Stage IV & $14(3.0)$ & $12(2.7)$ & $22(2.7)$ & $30(2.2)$ & \\
\hline
\end{tabular}

$S D$ standard deviation

Table 2 Surgical outcomes by surgeon experience

\begin{tabular}{|c|c|c|c|c|c|}
\hline $\begin{array}{l}\text { Surgeon experience (number of gastrectomies before } \\
\text { incident case) }\end{array}$ & $\begin{array}{l}<51 \\
n=450(\%)\end{array}$ & $\begin{array}{l}51-100 n=450 \\
(\%)\end{array}$ & $\begin{array}{l}101-200 n=814 \\
(\%)\end{array}$ & $\begin{array}{l}>200 n=1,346 \\
(\%)\end{array}$ & $P$ value \\
\hline Operating time $(\mathrm{min})$ & $214.0 \pm 62.4$ & $191.3 \pm 51.7$ & $178.6 \pm 61.0$ & $164.0 \pm 48.5$ & $<0.001$ \\
\hline Number of dissected lymph nodes (mean \pm SD) & $36.1 \pm 15.9$ & $37.4 \pm 16.4$ & $38.8 \pm 16.2$ & $42.3 \pm 16.2$ & $<0.001$ \\
\hline Residual tumor classification & & & & & 0.011 \\
\hline R0 & $438(97.3)$ & $431(95.8)$ & $802(98.5)$ & $1,296(96.3)$ & \\
\hline $\mathrm{R} 1$ & $12(2.7)$ & $19(4.2)$ & $12(1.5)$ & $50(3.7)$ & \\
\hline Duration of hospital stay (mean $\pm \mathrm{SD}$, days) & $13.0 \pm 8.1$ & $12.7 \pm 7.0$ & $13.1 \pm 15.2$ & $11.4 \pm 8.2$ & 0.001 \\
\hline Postoperative mortality & $4(0.9)$ & $6(1.3)$ & $5(0.4)$ & $6(0.3)$ & 0.23 \\
\hline
\end{tabular}

Effect of surgeon experience on the overall survival of gastric cancer patients

The more experience the surgeons had the greater was the overall survival of patients $[\leq 50$ versus $100<\leq 200$; hazard ratio (HR) $0.775,95 \%$ confidence interval (CI) $0.617-0.914, P=0.029]$. Figure 1a shows the 5-year overall survival based on surgeon experience. There was an improvement in survival with increasing surgeon experience up to 100 prior surgeon experiences, and no further improvement after that. Analysis of patients who underwent $\mathrm{R} 0$ resection only showed that there was a significant association between overall survival and surgical experience (Fig. 1b). These trends were shown in stage II, III, and IV tumors (Fig. 2a-d).

Univariate and multivariate analyses

Univariate and multivariate analyses showed that the ages of patients (HR 1.526, $95 \%$ CI 1.310-1.778, $P<0.001$ ), macroscopic type (HR 1.329, $95 \%$ CI 1.084-1.629, $P<0.001$ ), TNM stage (HR 2.241, $95 \%$ CI 1.511-3.322, 

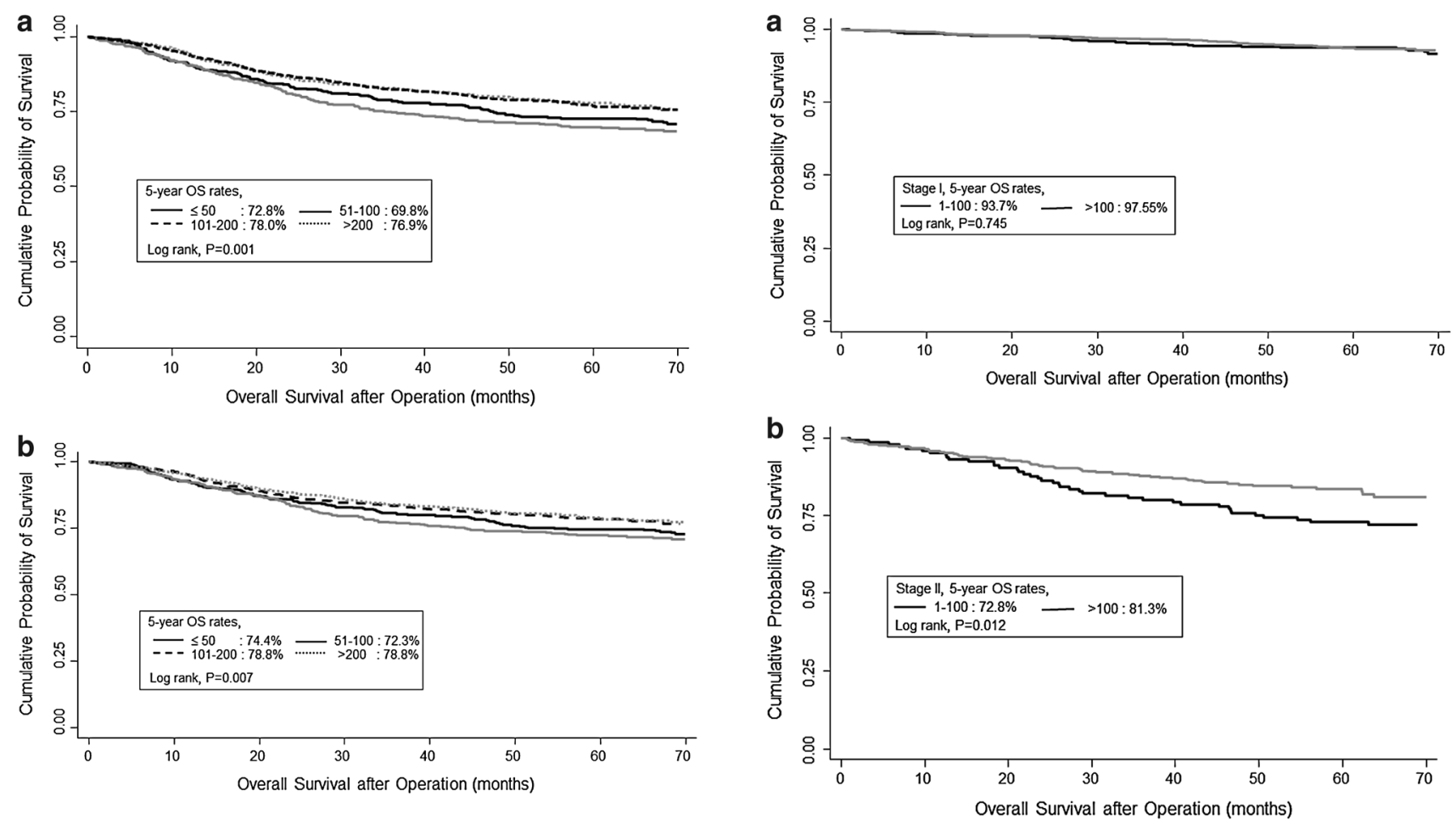

Fig. 1 a Survival curves by surgical experience for all patients. As surgical experience increased, the survival of patients improved. Overall survival (OS) rate at 5 years after 100 surgical experiences remained steady. b Survival curves based on surgical experience for patients who underwent R0 resection only. As surgical experience increased, the survival of patients improved. Overall survival rates here were higher than those of patients who underwent R0 and R1 resection in each period. Overall survival rate at 5 years after 100 surgical experiences was steady

$P<0.001$ ), and classification of residual tumor (HR 1.840, $95 \%$ CI $1.362-2.485, P=0.001$ ) were significantly associated with survival (Table 3). After adjusting for wellknown factors for survival including age, sex, tumor size, macroscopic type, and TNM stages, surgeon experience was a significant, independent risk factor for survival of patients with gastric cancer $(<51$ versus $\geq 201$, HR 0.797 , $95 \%$ CI $0.642-0.989, P=0.040)$.

\section{Discussion}

The main findings of the present study are (1) the overall survival after gastrectomy with $\mathrm{D} 1+\beta$ or more lymph node dissection increased as the experience of the operating surgeon increased; (2) overall survival does not steadily increase but decreased after 50 experiences and then steadily again increased after 100 prior experiences; and (3) short-term surgical outcomes including operating time, duration of hospital stay, and operative mortality seemed to improve as operative experience increased.
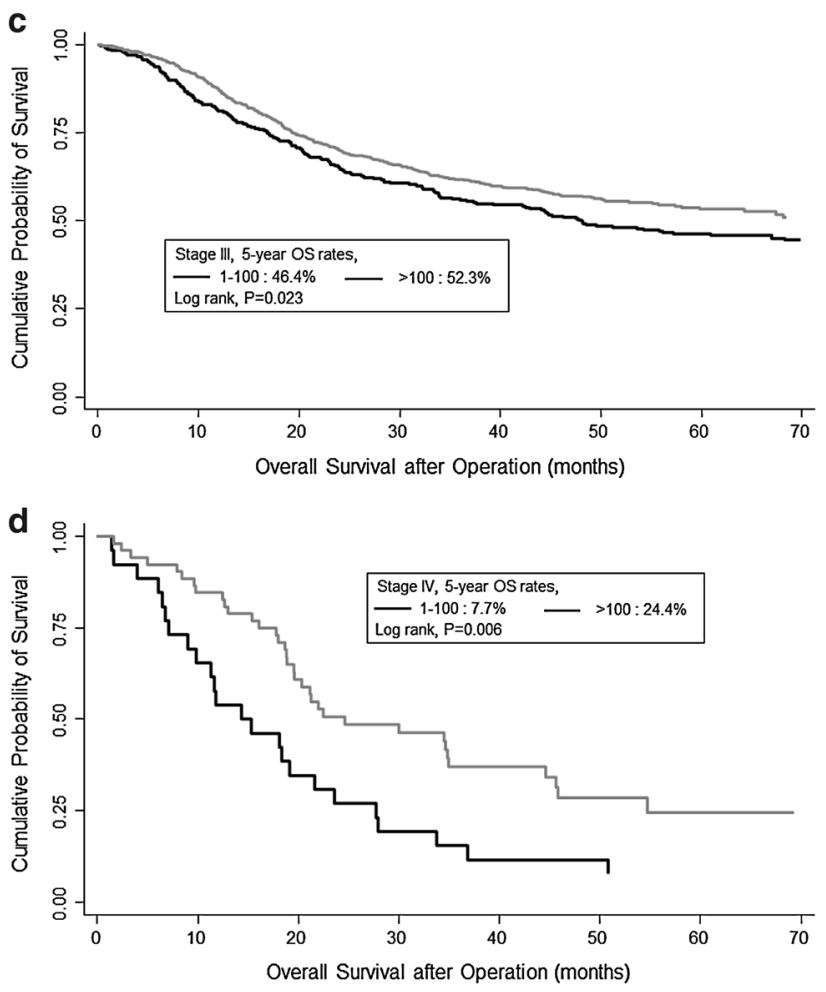

Fig. 2 a-d Overall survival (OS) curves based on surgical experience for stages I-IV, respectively. The 5-year overall survival rates after 100 surgical experiences were higher than those at 100 or fewer surgical experiences at each TNM stage except at stage I

A previous publication regarding the learning curve of gastric cancer surgeries that used mortality and morbidity rates as outcome measures suggested that the learning 
Table 3 Univariate and multivariate analysis for overall survival

\begin{tabular}{|c|c|c|}
\hline Characteristics & Crude hazard ratio ( $95 \%$ confidence interval) & Adjusted hazard ratio ( $95 \%$ confidence interval) \\
\hline \multicolumn{3}{|l|}{ Sex } \\
\hline Male & 1 & 1 \\
\hline Female & $0.854(0.726-1.004)$ & $0.907(0.767-1.073)$ \\
\hline \multicolumn{3}{|l|}{ Age (years) } \\
\hline$<60$ & 1 & 1 \\
\hline$\geq 60$ & $1.403(1.210-1.627)$ & $1.526(1.310-1.778)$ \\
\hline \multicolumn{3}{|l|}{ Location of tumor } \\
\hline Upper one third & 1 & 1 \\
\hline Mid one third & $0.640(0.521-0.786)$ & $1.111(0.889-1.389)$ \\
\hline Low one third & $0.616(0.513-0.739)$ & $1.376(1.081-1.752)$ \\
\hline \multicolumn{3}{|l|}{ Tumor size } \\
\hline$<4.2 \mathrm{~cm}$ & 1 & 1 \\
\hline$\geq 4.2 \mathrm{~cm}$ & $3.469(2.929-4.109)$ & $1.188(0.979-1.441)$ \\
\hline \multicolumn{3}{|l|}{ Type of gastric resection } \\
\hline Subtotal gastrectomy & 1 & 1 \\
\hline Total gastrectomy & $2.445(2.112-2.831)$ & $1.192(0.980-1.451)$ \\
\hline \multicolumn{3}{|l|}{ Borrmann type } \\
\hline $0^{\mathrm{a}}, \mathrm{I}, \mathrm{II}$ & 1 & 1 \\
\hline III, IV & $3.935(3.325-4.656)$ & $1.329(1.084-1.629)$ \\
\hline \multicolumn{3}{|l|}{ Differentiation of tumor } \\
\hline Differentiated & 1 & 1 \\
\hline Undifferentiated & $1.680(1.433-1.970)$ & $1.105(0.936-1.306)$ \\
\hline \multicolumn{3}{|l|}{ TNM stage } \\
\hline I & 1 & 1 \\
\hline II & $3.089(2.340-4.078)$ & $2.406(1.761-3.289)$ \\
\hline III & $9.855(7.867-12.346)$ & $6.443(4.801-8.647)$ \\
\hline IV & $22.323(16.054-31.042)$ & $10.730(7.047-16.337)$ \\
\hline \multicolumn{3}{|l|}{ Residual tumor } \\
\hline R0 & 1 & 1 \\
\hline $\mathrm{R} 1$ & $5.805(4.514-7.466)$ & $1.840(1.362-2.485)$ \\
\hline \multicolumn{3}{|c|}{ Surgical experience (number of operations) } \\
\hline$\leq 50$ & 1 & 1 \\
\hline $50 \leq 100$ & $1.124(0.885-1.427)$ & $1.059(0.824-1.362)$ \\
\hline $100 \leq 200$ & $0.775(0.617-0.974)$ & $0.788(0.624-0.996)$ \\
\hline$>200$ & $0.792(0.642-0.978)$ & $0.770(0.620-0.998)$ \\
\hline
\end{tabular}

${ }^{a}$ Borrmann type 0 represents early gastric cancer

curve in gastrectomy with D2 lymph node dissection ranges from 15 to 25 cases in a study including 38 patients [15]. This previous study focused primarily on the feasibility of D2 lymph node dissection, used a restricted sample size, and presented limited information on oncological outcome. However, in the case of cancer surgery, oncological outcome should be considered so that clinical trial results are correctly interpreted. Another report of the learning curve for total gastrectomy in patients with gastric cancer analyzed the number of retrieved lymph nodes as a main outcome in terms of oncological surgery. However, the endpoint of this trial still was not actual survival [15].
We observed that overall survival after gastrectomy with D2 lymph node dissection increases with surgical experience. This finding was consistent when we compared the overall survival in each of the TNM stages except stage I. Recent reports of prostate cancer surgery have shown that as a surgeon's experience increases, cancer control after radical prostatectomy improves [8]. However, differences in the mix of cases seen by surgeons might have contributed to residual confounding. Further, the endpoint of this study was biochemical recurrence, so the findings are of uncertain clinical relevance to patients. 
Although this study included patients with a predominance of early gastric cancers, overall survival after gastrectomy with D2 lymph node dissection increased with surgical experience. Improved quality of D2 lymph node dissection might be one reason because the number of retrieved lymph nodes was increased and operating times were decreased with surgical experience. Although two European randomized controlled trials (Dutch and MRC trials) that compared D1 and D2 lymph node dissection failed to show a benefit of D2 lymph node dissection, 10-year survival follow-up data have shown the beneficial effect of D2 lymph node dissection [5, 6].

Our study here is the first report using actual survival of cancer patients. We found that the overall survival was not steadily increased but decreased after the initial experiences and then steadily increased again. This blended pattern of survival might be caused by the selection bias of the surgeons themselves. After initial experiences (specifically, the initial 50 experiences), surgeons tried to do more aggressive surgeries. The highest postoperative mortalities and the rates of $\mathrm{R} 1$ resection after initial experiences might be the result of less selection of patients by surgeons. However, this selection bias seemed to be overcome as experience further increased.

Surgical experience is hard to assess. We used the number of operations as surgical experience. However, we can expect that as the number of operations is increased, much operation quality, including not only the number of dissected lymph nodes but also operating times, blood loss during the operation, and postoperative patient care, might be increased. We assumed that surgical experience is the sum of all those factors. Therefore, improved survival after 100 operations in this study might be results of systematic improvement in care of patients including operation skills.

The major limitation of this study is that it is a retrospective study and as such the treatment strategy was not based on random assignment. In other words, a selection bias for choosing treatment modality might have occurred. However, a randomized clinical trial for the comparison of survival based on a learning curve is not possible. Another limitation of this study was the limited data of date of recurrence. Therefore, we could not clearly show the relationship between experience and diseasespecific survival. This study analyzed data from highvolume centers in Korea and included surgeons who were trained for upper gastrointestinal surgery for at least 2 years. These strict inclusion criteria for surgeons and institutions might have influenced the results and might have reduced variation among surgeons. Postoperative chemotherapy might have also influenced patients' survival. Different chemotherapeutic regimens and indications of adjuvant chemotherapy from different institutions might have influenced survival of patients. Intervals between experiences might also influence results. Although high-volume centers were included in this study, the number of operations during the study period could not be the same. However, this study clearly showed a trend of learning curve in cancer surgery. During the study period, laparoscopic gastric cancer surgeries began to be done in Korea, especially by young surgeons. Experiences of D2 lymph node dissection in open surgeries might help adequate lymph node dissection during laparoscopic surgery, and the experience of laparoscopic surgeries might help to understand the anatomy of the stomach and thus help to raise the quality of lymph node dissection during open surgeries. Experiences of laparoscopic surgeries were variable among the surgeons included in this study, which might have influenced the results.

In summary, as a surgeon's experience increases, actual survival after gastric cancer surgery improves. Further research is needed to develop specific techniques to reduce the learning curve, including imaginary exercises or computer simulations. Interestingly, not the initial period $(<50$ experiences) but the second period (50-100 experiences) might be the most dangerous period, based on the fact that survival of patients was the poorest and postoperative morbidities and mortalities were the highest during the second period.

Conflict of interest No competing interests declared.

\section{References}

1. Jemal A, Bray F, Center MM, et al. Global cancer statistics. CA Cancer J Clin. 2011;61:69-90.

2. Sasako M, Sano T, Yamamoto S, et al. D2 lymphadenectomy alone or with para-aortic nodal dissection for gastric cancer. N Engl J Med. 2008;359:453-62.

3. Brennan MF. Lymph node dissection for gastric cancer. N Engl J Med. 1999;340:956-7.

4. Siewert JR, Bottcher K, Stein HJ, et al. Relevant prognostic factors in gastric cancer: ten-year results of the German Gastric Cancer Study. Ann Surg. 1998;228:449-61.

5. Schwarz RE, Smith DD. Extended lymph node dissection for gastric cancer: who may benefit? Final results of the randomized Dutch gastric cancer group trial. J Clin Oncol. 2005;10:5404-5.

6. Cuschieri A, Fayers P, Fielding J, et al. Postoperative morbidity and mortality after D1 and D2 resections for gastric cancer: preliminary results of the MRC randomised controlled surgical trial. The Surgical Cooperative Group. Lancet. 1996;347:995-9.

7. Begg CB, Cramer LD, Hoskins WJ, et al. Impact of hospital volume on operative mortality for major cancer surgery. JAMA. 1998;280:1747-51.

8. Vickers AJ, Bianco FJ, Serio AM, et al. The surgical learning curve for prostate cancer control after radical prostatectomy. J Natl Cancer Inst. 2007;99:1171-7.

9. Vickers AJ, Savage CJ, Hruza M, et al. The surgical learning curve for laparoscopic radical prostatectomy: a retrospective cohort study. Lancet Oncol. 2009;10(5):475-80. 
10. Sobin LH, Wittekind C. International Union Against Cancer (UICC) TNM classification of malignant tumours. 6th ed. New York: Wiley-Liss; 2002. p. 65-8.

11. Association Japanese Gastric Cancer. Japanese classification of gastric carcinoma, 2nd English edition-. Gastric Cancer. 1998;1:10-24.

12. Sakuramoto S, Sasako M, Yamaguchi T, et al. Adjuvant chemotherapy for gastric cancer with S-1, an oral fluoropyrimidine. N Engl J Med. 2007;357:1810-20.
13. Kaplan EL, Meier P. Nonparametric estimation from incomplete observations. J Am Stat Assoc. 1958;53:457-81.

14. Parikh D, Johnson M, Chagla L, et al. D2 gastrectomy: lessons from a prospective audit of the learning curve. Br J Surg. 1996;83:1595-9.

15. Lee JH, Ryu KW, Lee JH, et al. Learning curve for total gastrectomy with D2 lymph node dissection: cumulative sum analysis for qualified surgery. Ann Surg Oncol. 2006;13:1175-81. 